\title{
El impacto de los servicios públicos de cuidado a mayores en una zona rural de Extremadura
}

\author{
The impact of public caregiving services for elderly people in a rural area of Extremadura (Spain)
}

\author{
Chiara Cerri \\ Centro de Ciencias Humanas y Sociales. Consejo Superior de Investigaciones Científicas (CSIC). Madrid (España) \\ chiara.cerri@cchs.csic.es
}

\author{
NUEVAS RURALIDADES \\ MONOGRÁFICO COORDINADO POR SHARON R. ROSEMAN, SANTIAGO PRADO Y XERARDO PEREIRO
}

\begin{abstract}
RESUMEN
En este documento se presentan algunos de los resultados de una investigación centrada en las políticas públicas de cuidado, realizada en dos municipios de la Sierra de Gata, Mancomunidad de Extremadura en la provincia de Cáceres. Los datos obtenidos a través del trabajo de campo permiten sostener la importancia del desarrollo de los servicios públicos asistenciales como factor de cambio en las relaciones sociales y en los valores comunitarios de los habitantes de esta zona rural. En efecto, en los municipios de estudio, los servicios de asistencia para los mayores considerados dependientes han obtenido con el paso del tiempo una legitimidad socio-cultural por parte de la colectividad. De esta forma, en un proceso continuo, el cuidado formal y profesional se institucionaliza y legitima, al mismo tiempo que el cuidado familiar y el cuidado informal entre los vecinos residentes se transforma y disminuye. Esta dinámica conlleva entonces una mutación de las relaciones sociales en la comunidad y también un cambio en los tradicionales valores del cuidado.
\end{abstract}

\begin{abstract}
In this study, we present some of the results from a research project concerning public caregiving services conducted in two municipalities in Sierra de Gata (Cáceres, Extremadura, Spain). The information gathered through the fieldwork underscores the importance of the development of public welfare services as a factor in changing social relations and community values among the inhabitants in this rural zone. In effect, in the municipalities studied, services providing assistance to elderly individuals who are dependent have gained a sociocultural legitimacy within the local society. Therefore, through a continual process, formal and professional care have become institutionalized and legitimized. At the same time, care by family members and informal care between neighbours has been transformed and reduced. These dynamics illustrate the changes in social relations in the community and a change in the traditional values associated with care.
\end{abstract}

PALABRAS CLAVE

envejecimiento | cuidado | familia | vecindad | rural

KEYWORDS

ageing | care | family | neighbourhood | rural

\section{Introducción (1)}

En los últimos años se ha hecho cada vez más evidente que la sociedad española está encaminada hacia un proceso de envejecimiento demográfico a causa de, entre otras cosas, la mejora de las condiciones de vida que permite vivir más tiempo y la disminución de la natalidad. Conjuntamente, el continuo flujo migratorio que ha empujado y sigue empujando a los habitantes más jóvenes hacía otras localidades, hace que en las zonas rurales se genere una amplia presencia de población mayor.

Estos procesos caracterizan la realidad socio-demográfica de la zona de estudio, la Mancomunidad de Sierra de Gata en la provincia de Cáceres (Extremadura), donde en los últimos años las instituciones locales han puesto en marcha algunos servicios de asistencia para las personas consideradas dependientes, a través de los recursos del Sistema para la Autonomía y Atención a la Dependencia 
(SAAD) creado a partir de la Ley 39/2006, de 14 de diciembre, de Promoción de la Autonomía Personal y Atención a las personas en situación de dependencia (desde ahora Ley de Dependencia).

En el siguiente artículo se presentan algunos de los resultados de una investigación realizada en el 2010, centrada en el análisis del cuidado ofrecido a personas mayores, con el propósito de mostrar el impacto que las políticas públicas asistenciales están teniendo en las relaciones sociales, en particular de los más mayores y en los valores colectivos de la comunidad rural.

El interés de realizar la investigación en un contexto rural se debe, en primer lugar, al hecho de que los cuidados en zonas rurales han sido en general poco considerados por parte de las demás investigaciones, centradas más en los espacios urbanos. En segundo lugar, el actual proceso de sobreenvejecimiento poblacional (Alberdi Collantes 2007), típico de estas zonas, se configura como un interesante contexto para explorar más de cerca los vínculos, las percepciones y vivencia de los mayores.

Sin entrar en el más extenso debate sobre la distinción rural-urbano, nos parece necesario aclarar que, en este estudio, con "rural" se define un espacio socio-demográfico y geográfico limitado, caracterizado por una red socio-comunitaria con elementos y valores compartidos que determina unas particulares relaciones de cuidado, conceptualizando así la realidad rural más allá de sus características demográficas y económica.

Por otra parte, aunque en ámbito estadístico y administrativo se definen "mayores" aquellos que superan los 65 años, edad media de jubilación y por lo tanto, en términos económicos-monetarios, edad de salida de la población activa, en el estudio se recurre al término "mayores" para nombrar colectivamente los sujetos involucrados, teniendo conciencia de sus implicaciones hermenéuticas y sin pretender presentar un grupo totalmente homogéneo. De hecho, los principales informantes, en particular mujeres, superan de media los 75 años de edad, por lo que se debería hablar de una "cuarta edad" o de los "mayores viejos" para diferenciarlos de los "mayores jóvenes". Esto habría presupuesto considerar la edad cronológica como la variable más importante a la hora de elegir y presentar los sujetos de estudio. Al contrario, la intención ha sido atender las posiciones que asumían los mayores en el tema de los cuidados. De esta manera, se he intentado mantener un punto de vista que permitiera concebir los sujetos de estudio como subjetividades dentro de unos campos comunes, con sus elementos y valores colectivos, para llegar a tener una visión conjunta de la realidad respetando sus individualidades. De hecho, se ha intentado tener presente las diferentes condiciones socio-económicas de los mayores, pasadas y actuales, que presentan una gran variedad.

Finalmente, una nota aclaratoria. La mayoría de las personas a las que se refiere este trabajo son mujeres, por lo que, siguiendo un principio de economía del lenguaje, se ha optado por la escritura en femenino genérico con el objetivo de facilitar la lectura del texto y evitar posibles confusiones.

\section{Los servicios públicos de cuidado en el contexto de estudio}

La Comarca natural de Sierra de Gata está situada en el extremo occidental de la región de Extremadura. Limita, al norte, con la provincia de Salamanca y al oeste con Portugal. Su superficie total es de 1.258 $\mathrm{Km}^{2}$ y cuenta con 22.349 habitantes, distribuidos en 19 municipios, de los que el $27 \%$ son mayores de 65 años: el 15\% son mujeres y el $12 \%$ son varones (INE 2010).

El trabajo de campo se ha realizado en dos municipios de alrededor de 2.000 y 1.000 habitantes (2), que presentan unas características claves para el particular desarrollo de las políticas públicas de cuidado. Estas características son:

a) La proximidad, no tanto espacial debida a las restringidas dimensiones del asentamiento, cuanto la proximidad relacional debida a la antigua formación de los pueblos, que se va formalizando y modificando con el paso del tiempo. De hecho, es la cercanía relacional que permite la creación de una 
red socio-comunitaria con elementos y valores compartidos: se instauran unas específicas condiciones simbólicas y materiales que dan lugar a una regulación interna de la vida social, económica, cultural, política, etc. y sus relativas modificaciones.

b) El proceso de envejecimiento demográfico, debido en particular al aumento de la esperanza de vida; la emigración pasada y presente que ha dado lugar a un proceso de despoblación y, por consiguiente, de sobreenvejecimiento (Alberdi Collantes 2007) o envejecimiento migratorio (Pérez Díaz 2005); el retorno al municipio de origen de individuos que emigraron para trabajar y, una vez retirados del empleo, deciden volver al pueblo.

Una de las consecuencias del proceso de envejecimiento demográfico y de despoblación que interesa el sistema de cuidado es la condición de soledad de las mayores.

En primer lugar, soledad como tipología de hogar: en ambos municipios el $70 \%$ de la población con más de 65 años, en particular mujeres, vive sola o en pareja (INE 2001). No obstante, el estado material de soledad no se limita a la forma de convivencia, ya que, en muchos casos, se intensifica por la ausencia en el municipio de los hijos, las hijas y los demás familiares cercanos que han emigrado.

Dicha condición de soledad se contrapone a la cercanía relacional de los municipios, donde todos se conocen y la gente difícilmente está sola: siempre se reciben visitas en casa, aunque se trate del repartidor de butano, y en los paseos por el pueblo es imposible no encontrar alguien con quien intercambiar unas palabras. Sin embargo, el "no estar solo" no siempre equivale al "no sentirse solo": se puede estar físicamente en compañía, pero se puede seguir sintiéndose solo. O al revés, se puede estar solo y no sentirse solo. Se trata de distintas naturalezas de soledad y de distintas naturalezas de compañía. En este caso, la soledad emotiva y la ausencia de los familiares son las principales causas de malestar entre las mayores.

Aunque estén solas, no todas las personas mayores se encuentran en una situación de dependencia, ya que no existe una asociación lineal entre envejecimiento y dependencia (Vega Solís 2009). No obstante, desde las administraciones se considera que la situación de soledad "práctica" es el principal problema de las mayores, porque no pueden recibir el cuidado que necesitan por parte de sus familiares. Por lo tanto, en los últimos años se han desarrollado unos servicios públicos de asistencia para solucionar dicha situación de desamparo.

Como podemos ver en la tabla de abajo, ambas localidades cuentan con los servicios de Ayuda a domicilio, Teleasistencia y Comida a domicilio. Una de ella cuenta además con un Centro de día, que funciona exclusivamente como Comedor, y una Residencia concertada. En el otro municipio en cambio existen unos Pisos Tutelados, con una gestión externa, donde también hay el servicio de Comedor y de Lavandería para externos. 


\begin{tabular}{|c|c|}
\hline \multicolumn{2}{|c|}{ SERVICIOS PÜBLICOSDE ASSTENCIA A LA DEPENDENCIA } \\
\hline Teleasistencia & $\begin{array}{l}\text { Las usuarias tienen una centralita conectada al teléfono en casa, para } \\
\text { que los teleoperadores puedan llamar, y un medallón que deberian } \\
\text { llevar siempre al cuello, para conectarse con la central del servicio en } \\
\text { caso de emergencia, que sin embargo, en la mayoria de los casos, } \\
\text { dejan en un cajón o encima de la mesita de noche }\end{array}$ \\
\hline $\begin{array}{l}\text { Ayuda a } \\
\text { domicilio }\end{array}$ & $\begin{array}{l}\text { La aux iliar de Ayuda a domicilio trabaja unas horas a la semana } \\
\text { (máximo tres horas al dia todos los dias) en casa de las usuarias para } \\
\text { realizar servicios relacionados con la atención de las necesidades } \\
\text { domésticas o del hogar (limpieza, lavado, cocina, compra u otros) o } \\
\text { servicios relacionados con la atención personal (aseo personal, ayuda a } \\
\text { comer, etc.) en base a las necesidades de la persona determinadas por } \\
\text { la asistente social. }\end{array}$ \\
\hline Centro de dia & $\begin{array}{l}\text { Funciona principalmente como Comedor: una trabajadora prepara la } \\
\text { comida y las usuarias van a comer alli o, en algunos casos, recogen la } \\
\text { comida para llevarrsela a su casa. Además es desde aqui que sale la } \\
\text { comida a domicilio que se reparte entre varias usuarias. }\end{array}$ \\
\hline Residencia & $\begin{array}{l}\text { Edificio de nueva construcción a las afuera de la localidad donde } \\
\text { residen mayores considerados "ho validos" o "asistidos" las } 24 \text { horas } \\
\text { del dia, teniendo a disposición servicios continuados de carácter } \\
\text { personal (aseos, comida, etc.) y sanitario (enfermeria y fisioterapeuta) } \\
\text { y la posibilidad de salir del edificio, aunque son pocos los que salen a } \\
\text { causa de la lejania del centro del municipio. }\end{array}$ \\
\hline $\begin{array}{l}\text { Pisos } \\
\text { Tutelados }\end{array}$ & $\begin{array}{l}\text { Edificio a las afuera de la localidad donde residen mayores } \\
\text { considerados "validos" las } 24 \text { horas del dia, teniendo a disposición } \\
\text { servicios continuados de carácter personal (aseos, comida, etc.) y la } \\
\text { posibilidad de salir del edificio, que muchos aprovechan para darse } \\
\text { paseos. Además ofrece servicio de Comedor y Lavanderia para los } \\
\text { mayores no residentes. }\end{array}$ \\
\hline
\end{tabular}

En ambas localidades no hay más servicios de asistencia para mayores que estos. Las personas que contratan una asistente privada para el cuidado son casi nulos, una o dos en cada municipio. En cambio es común que los mayores, sobre todo mujeres, contraten una persona para realizar de vez en cuando una limpieza en profundidad de la casa, porque ellas solas ya no pueden y este trabajo no está incluido en las tareas de la auxiliar de Ayuda a domicilio. Este fenómeno explica en parte porque en esta zona no hay población inmigrante que se ocupe de las tareas de cuidado como suele pasar en las ciudades más grandes, ya que la limpieza de las casas se configura como el principal nicho laboral para las mujeres jóvenes "autóctonas".

Otro espacio utilizado para los mayores es el Hogar del Pensionista. En una localidad está situado en la planta de arriba del Centro de día-Comedor y en la otra se ha fusionado con el bar que está debajo de los Pisos Tutelados. Los Hogares del pensionista existen en los municipios desde antes de la implantación de los servicios de asistencia y funcionan un poco como los Centro de Mayores que hay en otras ciudades. Sin embargo, mientras que en los Centro de Mayores se realizan actividades como gimnasia, talleres de memoria, pintura, costura, etc., en la zona de estudio los Hogares son espacio que sirven más como cafetería-bar donde los mayores pasan el tiempo jugando a las cartas o al bingo.

Gran parte de los servicios públicos de asistencia se rigen por el copago, por lo que las beneficiarias pagan una parte del importe: alrededor de 3 euros por el Centro de día-comedor; de 3 a 6 euros por la Ayuda a domicilio, que va en base a sus ingresos; el $75 \%$ de su pensión para la Residencia y los Pisos Tutelados. 
En lo que concierne a las beneficiarias, en general van de los 65 a los 100 años, aunque la mayoría se sitúa entre los 75 y los 90 años. Hay también casos de personas de alrededor de 50 años con particulares necesidades que reciben Ayuda a domicilio, así como casos de mayores que sufren graves enfermedades y disfunciones, por lo que les es imposible seguir viviendo completamente solos en sus casas, que se establecen en casa de alguna hija o en una Residencia. Asimismo, las mujeres son las que reciben más servicios a domicilio, mientras que los varones son más numerosos en los Centros Residenciales, donde se instalan cuando quedan viudos y ya no tienen a sus esposas para que les cuiden.

Este fenómeno, por un lado, expresa la creencia de que cuidar es tarea prevalentemente femenina, porque las mujeres son más "adecuadas" para esto. Por otro, revela la existencia de un grupo de población, el masculino, que ha sido y sigue siendo en parte socialmente justificado y exonerado del cuidar. Esta exoneración social explica también porque algunas actividades de cuidado, como cocinar, planchar, limpiar, etc., no son reconocidas como trabajo de cuidado por parte de algunos: se trata de tareas que siempre han realizado las mujeres de la familia, y que siguen realizando, y se perciben como hechos cotidianos "normales".

\section{Formas, dimensiones y agentes del cuidado}

Los servicios de cuidado tienen como finalidad principal atender las necesidades en la realización de las actividades básicas de la vida diaria (ABVD), aquellas que una persona ha de realizar diariamente para poder vivir de forma integrada en su entorno habitual y cumpliendo su rol social (Rogero-García 2009), para mejorar la calidad de vida de las personas y también facilitar una existencia autónoma en su propio medio.

Este tipo de cuidado puede ser denominado "formal" cuando está realizado por personas retribuidas, profesionales o no, dentro y fuera de casa, o "informal": "un tipo de apoyo social que proviene de la red de apoyo de la persona y que a su vez deriva de su red social" (Rogero-García 2010: 36).

Sin embargo, la distinción entre cuidado formal y cuidado informal no corresponde del todo a la realidad, ya que, en la mayoría de los casos, las formas de cuidado se entrelazan entre sí dando lugar a situaciones intermedias (Comas D'Argemir y Roca 1996) como las "redes de cuidado": el cuidado se realiza por parte de los familiares, en ámbito doméstico y de forma no retribuida, y al mismo tiempo se combina con los servicios sociales, como la Ayuda a domicilio, o también con los servicios privados de otras personas.

Otra distinción es la de Durán (2002), que recoge distintos tipos de cuidado según el espacio en el que se integran. Según la autora, existen dos sistemas principales: el doméstico y el extra-doméstico. En el primero se encuentra el autocuidado, el cuidado realizado por familiares (residentes o no en el hogar) y el cuidado realizado en el hogar del asistido por personas no familiares, remuneradas o no. En el sistema extra-doméstico se encuentran los servicios públicos, los servicios de voluntariado, los servicios cooperativos o mutualistas y los servicios para la venta con ánimo de lucro.

También en el caso de las personas que proporcionan cuidados, es posible diferenciar entre cuidadores formales e informales. Con "informales" se definen aquellos que tienen un elevado grado de compromiso afectivo hacia la tarea de cuidar y una atención sin límites de horarios. Se trata principalmente de familiares, mujeres $\mathrm{y}$, en algunos casos, de amigos y vecinos. A veces se les denomina como "cuidadores principales" por asumir una responsabilidad casi total en el cuidado, diferenciándose de los "cuidadores secundarios".

Sin embargo, no existen solamente cuidadores "físicos". También son considerados cuidadores "indirectos" instituciones como el Estado, las obras sociales, el mercado, las organizaciones de voluntariado, etc., en tanto que igualmente responsables del bienestar de la personas necesitada de 
cuidado.

Estas distinciones se relacionan con las diversas dimensiones del cuidado (Tronto 2005, 2010): por un lado el caring for (o taking care of), que indica la ayuda práctica, material, que algunos agentes dan a otros, un "ocuparse" de alguien que implica asumir la responsabilidad del trabajo que hay que hacer para cuidar. Por otro lado, el caring about, que se refiere más a dar y recibir cariño, afecto, apoyo moral y psíquico, un "preocuparse" de las personas necesitadas que implica el reconocimiento de la necesidad de cuidar (3). Para el análisis es importante realizar distinciones entre las diferentes dimensiones del cuidado, en particular entre el cuidado emocional y el cuidado práctico. Sin embargo, es fundamental considerarlas siempre como dimensiones relacionadas entre sí, ya que el cuidado es una actividad que tiene en consideración todos los aspectos de la vivencia de las personas, no sólo sus necesidades físicas, y se puede concebir como "tridimensional": es material, moral y emotivo (Martín Palomo 2008b).

Resulta entonces difícil definir de forma concreta qué es el cuidado, ya que asume un valor social no de por sí, sino en base a la percepción que se tiene de ello y su integración en determinadas relaciones sociales (Comas d'Argemir 1993). En algunos contextos puede ser considerado "empleo" o "trabajo", en otro simplemente "apoyo" y/o "asistencia". En general, se pueden englobar bajo el término de cuidado todas aquellas actividades que se realizan para el bienestar físico, psíquico y emocional de las personas (Comas d'Argemir 1993) y que suponen una interdependencia entre todos los agentes en juego, considerados dependientes y receptores de cuidado por naturaleza.

En los municipios estudiados, la cuestión del cuidado, de quién cuida a quién y quién quiere ser cuidado o no, se ha hecho más visible a causa del envejecimiento de la población y la consecuente situación de soledad "práctica" de las personas mayores. Por este motivo, como he mencionado anteriormente, las instituciones han instaurado unos servicios de asistencia que, a lo largo del tiempo, han obtenido reconocimiento por parte de la población hasta alcanzar una legitimidad socio-cultural. En particular los servicios que permiten a la usuaria quedarse en su casa: Ayuda a domicilio, Centro de día, Comida a domicilio, Teleasistencia.

Pero antes de hablar de los cambios que ha introducido la institucionalización del cuidado en los valores colectivos, resulta interesante detenerse un momento en los cambios que se han introducido en el ámbito de empleo.

En efecto, en el caso de las políticas asistenciales ha aumentado la demanda de empleo en los servicios de enfermería, fisioterapia y comedores. Al mismo tiempo, se han formalizado nuevas figuras profesionales como las auxiliares de Ayuda a domicilio.

En este caso concreto ha crecido la oferta de cursos para la formación profesional de los habitantes locales y los ayuntamientos, para hacer frente a una situación de desempleo constante, han desarrollado un plan de empleo "rotatorio"(4): cada año se finalizan los contratos de trabajo y, a través de una nueva convocatoria pública y sucesiva selección, se asumen nuevas profesionales para la Ayuda a domicilio. De esta forma, se asegura a la población local interesada el trabajo continuado al menos durante un año.

En el caso del Centro de día-comedor las trabajadoras no tienen ninguna formación específica, mientras que en la Residencia y en los Pisos Tutelados las auxiliares realizan previamente cursos de especialización y prácticas. Se trata de curso, como en el caso de las auxiliares de Ayuda a domicilio, fomentados por los mismos Ayuntamientos o por la Comarca, la Provincia o la Comunidad de Extremadura.

Asimismo, la mayoría de las trabajadoras son originarias de las localidades donde trabajan, o viven en localidades cercanas. Esto significa que en algunos casos mantienen una relación vecinal o familiar con las usuarias. Sin embargo también hay trabajadoras, sobre todo las auxiliares de Ayuda a domicilio, que son originarias de otras localidades de Extremadura y que se han mudado hace unos pocos años por la mayor oferta de trabajo en la Sierra de Gata.

El hecho de que en todos los servicios de atención a personas mayores trabajan casi exclusivamente 
mujeres, revela tres fenómenos distintos:

a) El fomento de la incorporación de las mujeres al mercado del trabajo remunerado.

b) La institucionalización y profesionalización del cuidado y su salida de la esfera doméstica.

c) La reafirmación que el cuidado sigue siendo una actividad femenina, incluso más en estos municipios donde persiste la idea de que las mujeres son más "adecuadas" para cumplir las tareas del cuidar:

“¡No!... esto suele ser sobre todo... empleo femenino, [los hombres] ni se presentan ni... na'... ¡No! Los chicos mira... por ejemplo aquí tenemos un proyecto que ahora... el proyecto Isla... que es... Inserción... bueno, Isla sería Inserción Laboral, ¿no? Es un proyecto de Diputación... entonces aquí se echaron, al principio se dio publicidad... la gente se apuntaba a los cursos que más o menos le pudieran interesar... y lo que era, el... trabajo, con personas dependientes o con personas... en Residencias, sí que se apuntaban los chicos, ALGUNOS, no mucho. Claro, por el tema de poder ser celadores o... más por el tema de celadores más que de cuidado ya directo con el anciano, ¿no?" (Trabajadora servicios públicos)

Que el cuidado siga siendo una cuestión femenina lo revela también el hecho, en primer lugar, de que en las Residencias y Pisos Tutelados los hombres ocupan puestos de gestión o de alta profesionalización, como gerente o fisioterapeuta; o por el contrario, puestos de mantenimiento y vigilancia. En segundo lugar, los hombres constituyen las "nuevas" figuras profesionales, como dinamizadores deportivos, monitores de nuevas tecnologías, terapeutas ocupacionales, etc., implantadas a través de las políticas de envejecimiento activo (5). No solamente se mantiene la idea del cuidado como labor femenina, sino que se mantiene una división del trabajo asentada en los tradicionales roles de género.

No obstante, a pesar de la persistencia de la tradicional división del trabajo entre hombres y mujeres, es necesario subrayar que las necesidades derivadas del sobreenvejecimiento demográfico no tienen porque representar sólo "problemas" a los que hay que encontrar solución. También pueden resultar una ocasión para crear empleo y dinamizar, innovando, el panorama laboral de la población activa.

Se trata entonces de un cambio, debido a la institucionalización del cuidado, beneficioso para estas localidades tradicionalmente golpeadas por el desempleo y la emigración.

\section{El cuidado entre servicios públicos y familia}

La familia, sobre todo en las sociedades mediterráneas como España, sigue configurándose como el agente de asistencia predominante (Durán 2002, Bazo 2008). Y en la familia la relación de cuidado casi nunca es libre, sino que está determinada por un entramado de normas y valores que se basan en general en la responsabilidad y la obligación moral (Narotzky 2009, Cerri y Alamillo-Martínez 2012).

Sin embargo, ahora que el sistema de ayuda familiar típico de la sociedad española está pasando por un periodo de transformación, entran en crisis aquellas reglas socio-culturales no escritas que formaban sus fundamentos, variando considerablemente los valores y los lazos de parentesco (Conels 1996).

En los municipios de estudio, las personas mayores que residen solas o con algún familiar se han ido concienciando sobre la posibilidad de obtener cuidados prácticos por parte de los servicios públicos: si no pueden cocinar se apuntan al Centro de día para comer, para los recados y la limpieza tienen la ayuda de la auxiliar a domicilio, etc.

Los familiares también se benefician de las prestaciones públicas en caso de no poder asumir el cuidado de sus mayores, sintiéndose además respaldados y justificados por esto. Como es el caso de los parientes que viven fuera, que admiten que se van más tranquilos del pueblo porque sus padres están 
También es el caso de familiares que, aunque vivan en la misma localidad, prefieren no asumir el cuidado directo de sus mayores. En general esto se debe a conflictos que la convivencia con el mayor provoca o ha provocado en el pasado, como el caso de una señora que no quiere cuidar de su suegra, cosa que le "tocaría" ya que esta no tiene hijas y ella es la única nuera que vive en la misma localidad, a causa de incomprensiones pasadas. Otro ejemplo es el caso de un hijo único que se ha visto "obligado" a llevar su madre a los Pisos Tutelados porque esta no le permitía tener vida propia con su mujer y sus hijos, pidiendo continuamente su atención, chantajeándole emotivamente si él no respondía a sus demandas.

Se trata de situaciones conflictivas donde la presencia de los servicios públicos permite también a estos familiares, que tienen los "requisitos" para cuidar pero no quieren hacerlo, sentirse respaldados y justificados.

En general, a pesar de la heterogeneidad de las situaciones, ha habido una modificación en las relaciones familiares de cuidado gracias a la introducción de los servicios públicos, que ha dado lugar a una redefinición de la noción de "deber filial" (Bofill 2006) o responsabilidad y reciprocidad intergeneracional.

Sin embargo, esta situación se relaciona con el sentimiento de soledad que experimenta gran parte de las mayores, ya que el hecho de poder beneficiarse de unos cuidados prácticos a través de la Administración hace que soliciten a sus familias más cuidados emotivos. Lo único que les piden es consideración. Si esta no se recibe, o no se recibe de la manera que gustaría, las personas mayores se sienten abandonadas y "enferman" de soledad.

Por otra parte, también es cierto que hay una parte de la población residente que sigue cuidando directamente, y a tiempo completo, de sus familiares mayores. En estos casos, que se dan sobre todo cuando las cuidadoras no tienen empleo remunerado, se mantienen algunos valores tradicionales por los que el cuidado es asumido como una carga y una labor que hay que desempeñar por obligación.

La mayoría de las informantes que atendían a algún mayor manifestaban un sentimiento de culpabilidad simplemente al pensar en el no cuidar. Por el mismo motivo, no comprendían del todo las razones que llevaban a las demás mujeres "con tiempo libre" a no ocuparse de sus padres.

Estas incomprensiones no se deben solo al sentimiento de obligación familiar, sino que se vinculan también con los afectos que se desarrollan en la familia: cuidar de una persona se configura a veces no sólo como un compromiso, sino como un gesto de cariño.

"Es que mi madre se fue... se fue apagando apagando apagando... y eso es durísimo, porque mi madre tenía una lucidez... muy... y era una persona muy inteligente, muy culta... bueno, entonces... el que se vaya volviendo como... pfff... en el sillón... y menos mal que ha sido (su muerte), muy rápido." (mujer, 60 años).

El conflicto entre cuidados familiares y cuidados institucionales, entre los valores arraigados y los más actuales, se manifiesta en particular en el caso de las Residencias y los Pisos Tutelados. Su aprobación colectiva es todavía bastante débil, sobre todo a causa del valor y de la significación que las personas dan al espacio doméstico como fuente de autoridad y autonomía. De momento, el único espacio que permite a las mayores conservar dichas condiciones es su casa, porque saben que allí son las dueñas y pueden elegir lo que hacer y no hacer y también pueden decidir lo que los demás pueden o no pueden hacer.

"Es que esto es un pueblo pequeño, y una vez que se meten aquí (en Residencias), es porque los hijos no quieren saber cuenta de ellos. También a lo mejor los padres tienen la culpa, porque no vamos a decir todo de los hijos. Igual los padres también quieren vivir independientes o... yo qué sé. ¡Son rebeldes! y... 'Aquí mando yo', y eso ya, claro... las personas mayores, nos ponemos... nos 
ponemos un poco... un poco...pesaos. Yo creo que nosotros ya cuando nos toque... vamos a ser diferentes, ya tenemos otra mentalidad a lo mejor... no lo sé, nadie nos tiene que mandar, lo hacemos nosotros solos." (Se ríe) (hombre, 75 años).

Casi nadie quiere ingresar en una Residencia, considerada manifestación práctica de abandono, porque ahí tendrían menos poder. Es verdad que hay casos en que a las personas mayores no le queda otro remedio que irse a vivir en una Residencia o unos Pisos Tutelados, pero en general se resisten hasta el último momento. Los Centros Residenciales (Residencia y Pisos Tutelados) representan, para las personas mayores, la pérdida total de autoridad y autonomía y simbolizan el abandono y el aislamiento. Para algunos familiares, expresan desinterés total y hasta egoísmo.

"La Residencia llevará dos años, ¿no? De todas formas... con él... lo que a mi me han enseñao... lo que yo he vivido... yo no hubiera llevado jamás a mis padres. Si yo hubiera tenido, que yo comprendo, ¿no?, personas que tengan que trabajar, no pueden. ¡Es imposible! Pero yo, que me dedico... yo soy ama de casa, la típica maruja, pues oye me toca... (...). El otro día mismo, hace... nada, estaba, un caso... de una señora que está en la Residencia, y... mmm... tiene varias hijas y una de ellas es amiga mía desde... siempre. Bueno, pero hace que no la veo... ahora se quedó viuda también el año pasao y la pobre... no levanta cabeza, entonces, no sé como comentaron, pero digo ‘yyy... fulana, ¿qué tal la madre?', dice 'Está en la Residencia', digo 'Pero si no tiene nada que hacer'... no trabaja... los hijos ya... uno estudia en Málaga, el otro está en Salamanca o sea que ya no están... no tiene nada que hacer ¿cómo mete a su madre en una Residencia? O sea, lo hemos comentao como algo... RARO en nosotras. ¿Cómo se le ocurre meter a su madre en la Residencia si ella no tiene nada que hacer? 'Ya, claro... porque la hermana de Francia no quiere venir...claro, ella sola, no sé qué...'. (...) Pero suena... lo que te quiero decir es que suena raro, o sea que a nosotras, lo hemos comentao como algo... tendrá que meter a su madre en la Residencia..... Ay tal, que la pobre desde que se murió su marido no levanta cabeza'. Pues si a lo mejor le venía bien estar entretenida y tal y cual... no sé. O sea... a mí no me... cabe en la cabeza. Y a la otra amiga tampoco le cabía en la cabeza, ¿no? (...). También yo creo que es un poco, por lo que comento con las amigas y todas estas cosas, yo... a estas edades todas tenemos, los que tiene padres los tienen mayores, ¿no?, pues entonces hay de todo. Las hay que trabajan, las hay que no trabajan, las hay que piensan de una manera, las hay que piensan de otras. Hay familias que se están desplazando, dos o tres meses, cada hijo, aquí a estar con sus padres... y luego vuelve otro hijo, se van ellos y viene otro... hay familias que no, que se llevan a los padres... y hay familias de las que le meten en una Residencia viviendo aquí. O sea... hay de todo, ¿no? Entonces yo, por lo que hablo y tal, digo, es que yo creo que un poco también... los valores que te han transmitido tu familia... en... mi casa se le ha tenido mucho respeto hacia las personas mayores... era un respeto. No sé, era... era una cosa..." (mujer, 60 años).

"(¿Hay gente que ha vuelto al pueblo una vez jubilada y cuida de sus padres más mayores?). Hombre, yo tengo la desgracia de que ya no tengo ninguno. Pero bueno, hay jubilados que los tienen todavía, claro. Normalmente en los pueblos se hace así, se cuida en casa... Normalmente... pero hay mucha gente mayor en los Pisos también... es que... eso ya es más complicao; hay hijos que quieren cuidar a los padres, y hay hijos que no quieren saber nada de lo padres. Eso empezando por allí. Y luego... pues... ocurre que... a una persona mayor, de... de estos nuestros padres, a muchos meterles en una Residencia es... quitarle un montón de años de vida... las Residencias y además, demostrado. Usted no verá nunca, nunca, personas muy mayores, muy ancianos en las Residencias, se suelen morir antes. Analizando... vamos a ver, coge una persona de ochenta años y la mete en una Residencia y a los cuatro, cinco, seis años, cae (...). Yo creo que cuando los meten aquí ya no los llevan... yo ya... se acostumbran a tenerlos aquí... una vez que los meten aquí ya... aquí... cuando los hijos se desentienden de los padres, estando bien, cuanto más ancianos peor" (hombre, 75 años).

Esta visión de los Centros Residenciales muestra cómo en el cuidado se contraponen valores nuevos con valores antiguos: por un lado la legitimación de los servicios públicos, la profesionalización del cuidado, la autonomía del cuidador, etc. Por otro lado, la responsabilidad y el respeto hacia las personas 
mayores, la reciprocidad intergeneracional y los afectos más emotivos.

Esto se debe a que no existe una moralidad universal e invariable del cuidar, sino que esta se configura en base al contexto y la situación en la que los actores se encuentran. Un "situacionismo moral" (Lisón Tolosana 2010: 89) que hace que un mismo acto, un mismo cuidado, se califique de manera distinta según actores, edad, momento, espacio, etc., dentro de una ética del cuidado, como teoría moral contextual, donde "la imaginación moral, el carácter y las acciones deben responder a la complejidad de una situación dada" (Tronto 1987: 658).

En este caso, ha sido la observación participante de las vivencias cotidianas de los sujetos que ha permitido descubrir los valores en juego (Sanmartín 1999, 2003) en las relaciones de cuidado, donde tiene relevancia también la vecindad, tan importante en los municipios estudiados.

\section{Cambios en las relaciones vecinales}

En ambos municipios, los lazos entre vecinas son de por sí complejos a causa de enemistades, envidias, miedo a los cotilleos, etc., sobre todo entre mujeres. Por este motivo, muchas personas mayores han reducido los contactos entre sí y han preferido quedarse solas o restringir su grupo de amistades. Aunque se trate de personas que viven solas en sus hogares, en la mayoría de los casos se detecta un rechazo hacia la realización de actividades comunes como ir a jugar a las cartas, estar en la calle charlando con las vecinas, hacerse visitas en las casas, etc.

En una visita a su casa, una mujer de 78 años que vive sola con el hijo enfermo, reconoció que hace mucho que dejó de ir a misa porque quiere evitar los cotilleos de las demás mujeres. Por el mismo motivo no quiere que nadie suba a su casa, ni ella tiene interés en ir a casa de las vecinas.

Otra mujer mayor, durante una conversación informal debajo del portal de su casa, dijo que con unas vecinas que viven al final de su calle, entre las cuales está su cuñada con quien se lleva mal, no quiere nada. Ni siquiera tiene interés a entretenerse con ellas jugando a las cartas porque, como ella misma puntualizó, no le gustan las cartas ni la compañía. Las envidias, las enemistades del pasado y/o el miedo a las críticas son en esos casos más fuertes que la necesidad de compañía.

Esto se da sobre todo entre mujeres, ya que los hombres, en general, participan más en actividades comunes, como pasar la tarde en el bar jugando a las cartas.

Esta diferente postura entre hombres y mujeres se explica por la diferenciación de los roles de género: los hombres en general no cuidan, ni "se cuidan", ni se ocupan de la casa y, sobre todo en el caso de los mayores, siempre han tenido más libertad de movimiento en relación a los que está socialmente permitido y lo que no. Al contrario, las mujeres se ocupan de la casa, del cuidado de los suyos y están sometidas al juicio de los demás por lo que hacen o dejan de hacer: si se ocupan bien de la casa y de su familia, si se "portan bien", etc. Por lo tanto, hablando de las relaciones vecinales, es necesario tener en consideración el particular sistema de género del contexto de estudio, donde permanece una clara distinción entre hombres y mujeres, más visible entre las personas mayores, que afecta todos los aspectos de la colectividad.

"Porque los pueblos ¿lo que pasa? luego... ji ji ji ji... (Hace como imitando el ruido de los cotilleos) y yo eso es que ME REVIENTA! (...) en Barcelona... ahí es otro ambiente... otra cosa... ahí nadie se mete con nadie... pero aquí... ¡Madre mía por Dios!... aquí en esta calle... se ponen pa' ahí unas cuantas...dale, digo 'iToma! el Concilio Vaticano! ya está ahí puesto!'... pasa cualquiera... 'Mira, esa es... mmm... de la otra manera... y...', yo estoy en mi casa, ahora ya porque hace hoy... fresco, pero abro ahí... y yo todo lo que están hablando... gracias a Dios el oído lo tengo muy sano... todo lo que están hablando... lo oigo yo aquí sentada. ¡Y en la calle! En la calle... pero ¿no tendrán que hacer cosas? (...). ¡POR Mí... no critica nadie, y a mi... NADIE!, porque yo llevo las cosas muy rectas, que de torpe no tengo nada! reconozco todo bien... es que estos pueblos... yo no se como... serán 
otros, que la gente es muy envidiosa, ¿eh?, No sé... muy envidiosa... si sales porque sales, si entras porque entras... si... te compras ropa porque te la compras, si vas bien preparada porque vas..." (mujer, 74 años).

El miedo al "qué dirán" está demostrado también por la "costumbre" de mantener secretos acerca de lo que se hace o se dice, fenómeno que revela el peso de los cotilleos en las relaciones sociales. Como me dijo un día una mujer mayor "aquí en el pueblo, todos criticamos".

Se demuestra así que la vecindad no tiene un peso específico en las relaciones de los mayores si no está acompañada por la amistad. Es decir, la vecindad no es sinónimo de amistad (6), porqué, a diferencia de esta, no se basa en la voluntariedad de ambas parte (Cucó 1995).

Por lo tanto, la compañía física adquiere importancia solamente si tiene el valor añadido del afecto, lo que, además, permite superar el obstáculo de las envidias y los cotilleos. De hecho, hay casos de mujeres que mantienen relaciones de amistad con algunas vecinas. Sin embargo, se trata de casos muy puntuales que, la mayoría de las veces, interesa dos únicas "amigas". En general, las relaciones de amistad auténtica parecen muy escasas.

Aunque algunos estudios (Alberdi Collantes 2007, Tobío et ál. 2010) afirman que las personas mayores residentes en zonas rurales, a diferencia de aquellas que viven en entornos urbanos, tienen menores problemas de soledad gracias a las relaciones y ayudas vecinales, en el caso concreto de los municipios estudiados se está produciendo lo contrario: un mayor distanciamiento entre las vecinas. Parece que el motivo principal de esta transformación de los vínculos es el no querer "pedir favores".

El "pedir favores" se inserta en una relación de reciprocidad "obligada", donde, como dijo Mauss (1971), el carácter voluntario del don es "aparentemente" libre y gratuito: recibir dones comporta peligro, porque crea la obligación de devolverlos (Conels 1996). Por tanto, la demanda de un favor, o una ayuda, está directamente vinculada a la devolución del mismo. Las vecinas evitan entrar en este círculo de favores, utilizando los servicios públicos para recibir asistencia. De esta forma, por un lado contribuyen a que los servicios públicos adquieran cada día más legitimidad social; por otro lado, este proceso hace que las relaciones entre vecinas se deterioren aun más.

"Ay, no, por... huy, ¡las vecinas! si tienen... tengo que ir a la farmacia o eso... pues sí, dicen ‘¿Tienes que... traer algo?', pero no me gusta a mí darle quehacer a nadie” (mujer, 74 años).

No se puede negar que se dan casos en los que a algunas vecinas se les piden pequeños favores. Pero estas situaciones se dan por diferentes motivos:

a) Porque se trata de acciones que no requieren gran sacrificio: "ya que voy a comprar el pan para mí, lo compro también para la vecina".

b) Porque la vecindad está acompañada de la amistad y/o de lazos familiares no consanguíneos: comadres, ahijadas, etc. Se trata de casos de reciprocidad "generalizada" (Sahlins 1976), donde la incapacidad de responder a la obligación de devolver no siempre determina la interrupción de la relación y de la asistencia.

c) Porque los lazos familiares y/o de amistad expresan la existencia de una reciprocidad "aplazada" (Rogero-García 2010): la persona mayor ha ofrecido sus ayudas en el pasado y ahora solicita la devolución, o esta se le ofrece voluntariamente. Existe todavía reciprocidad porque se trata de algo que empezó en el pasado y que hay que continuar.

Sin embargo, en el caso de las demás vecinas, se utilizan los servicios públicos para suspender el círculo del intercambio o directamente no empezarlo.

Sahlins (1976) distingue tres formas de reciprocidad. La "generalizada" se da en la familia y se caracteriza por unas transacciones "por lo menos putativamente altruistas" (Sahlins 1976: 243), no 
solamente materiales, donde la obligación de devolver queda indefinida.

La reciprocidad "equilibrada" se encuentra fuera de la familia, o con parientes lejanos, y se caracteriza por un intercambio directo donde la obligación de devolver es definida en tiempo, cantidad y calidad, y cuando alguien se retracta, la relación termina.

La última, es la "negativa", un intercambio que tiene como objetivo "obtener algo gratuitamente: transacciones iniciadas y enfocadas hacia una ganancia utilitaria neta" (Sahlins 1976: 244), lo que podría se un fraude o un robo.

En el caso de estudio, la característica más apreciada de los servicios públicos es que escapan de la reciprocidad "altruista" o "equilibrada" de la vecindad, porque se basan en un intercambio económico: recibo un servicio porque pago. Este tipo de relación, de reciprocidad monetaria y utilitarista, que Sahlins no parece contemplar en su ensayo, se acaba en el momento mismo en que se corta la transición económica: si no pago, no recibo el servicio. Por lo tanto, la persona puede finalizarlo cuando quiera sin sentirse obligada a devolver "favores".

La importancia del dinero, además, se denota en los discursos de las personas mayores. Todos los usuarios recalcan mucho el tema de que ellos pagan los servicios, que, aunque sean baratos, no son gratis. Parece que quieren dejar claro que tampoco están recibiendo algún tipo de caridad, cosa que, como ya decía Mauss "es hiriente para quien la acepta" (Mauss 1971: 246).

La función de las vecinas se ha ido quedando entonces en un "estar ahí por si acaso". Más que ofrecer un cuidado directo y continuado, representan una seguridad que la gente tiene por si pasa algo; como último recurso al que no se recurre cotidianamente, sino en casos puntuales.

Esta situación se encuentra a "ambos lados" de la vecindad. Aquellas que no necesitan cuidados prácticos asumen la misma perspectiva: están dispuestas a echar una mano, pero son conscientes de que, en cualquier caso, existe la ayuda de los servicios públicos. Y de la familia, aunque viva lejos.

"Siíí... y aquí también habrá personas mayores que están solas. Aquí las hay. Pero es que... esa gente tiene también la Ayuda a domicilio que... que mete el Ayuntamiento (...). Ahora mismo nosotros hemos tenido ahí a una vecina que ya la han llevao a... tiene sus sobrinos, tenía un hermano todavía. Lo que pasa es que estaba... pues, más o menos... yo ahora mismo le he estao trayendo el pan... to' el tiempo, que... la mujer... bueno!... y el caso, pues bueno, que ya no estaba bien... y al final, han venido sus sobrinos que son los que tenían que venir, claro" (mujer 65 años).

Otro elemento característico de los servicios de cuidado en estos municipios es la cercanía entre instituciones y usuarios que permite la constitución de vínculos más íntimos y personales. Así, se introducen en los cuidados formales unos aspectos más emotivos que conectan el caring for con el caring about (Tronto 2005, 2010).

Algunas trabajadoras tienen una relación de confianza con algunas usuarias mayores y, sobre todo las auxiliares de la Ayuda y la Comida a domicilio, entran en sus casas sin llamar a la puerta y a veces sin que haya nadie dentro, realizan algunas tareas, etc. Esto demuestra hasta qué punto los mayores han incorporado los cuidado públicos en sus vidas, ya que esta "intrusión" de las trabajadoras en sus casas contrasta con el rechazo de dejar entrar las vecinas para preservar la propia intimidad.

Por otra parte, esta situación revela como la "profesionalidad" asociada a las trabajadoras, representa para los mayores un elemento de "protección" de posibles cotilleos.

Además, por esta cercanía y confidencialidad, las personas mayores recurren a las empleadas públicas por motivos que sobrepasan sus funciones. Piden auxilio para hacer funcionar un teléfono móvil o programar la tele, incluso utilizan sus servicios para charlar un rato y desahogarse. La cercanía y el contacto frecuente aumentan entonces la confidencialidad y el cariño entre trabajadoras y usuarios, y esto permite una aún mayor legitimación de los servicios públicos. 
"Sí, y vienen la chica y... y me limpia pa' aquí pa' allí... viene todas las mañanas una hora, y cuando, siempre no voy a limpiar porque yo no ensucio nada, y... le digo 'Siéntate, que me des compañía un ratito', y charlamos y eso me vale a mí mucho también. Y... y la mando al... al comercio o... a donde sea y va, me ayuda" (mujer, 89 años).

"Es día por día, día por día, día por día... y si tienen algún problema te lo cuentan... y si compra uno el móvil te lo trae pa' que se lo ponga. Digo ‘Fíjate! día por día aquí...', digo ‘¡Madre mía!', entonces están hechos a... y tú a ellos... entonces cuando falta uno... yo lo paso muy mal... muy mal, muy mal... porque les coges mucho cariño..." (trabajadora servicios públicos).

Así que, los servicios sociales han sido incorporados en la vida cotidiana de la población y más aún en la de las personas mayores. Por este motivo hablo de legitimación de los servicios públicos de cuidado, porque han llegado a ser parte del sistema social y cultural de los municipios, confirmando su existencia.

Para obtener legitimidad socio-cultural, cualquier elemento tiene que ser integrado en la vida de la población hasta devenir algo cotidiano y ordinario. Es lo que ha pasado con los servicios públicos de asistencia. Ahora son parte de la cotidianeidad, pero hasta hace 6 o 7 años no existían y su aceptación por parte de la población mayor ha sido larga y complicada.

“Entonces eso sí nos ha costao, ¿eh? Sí. Hasta que han ido entrando... hay de todo (...). Que no por que estén fuera de sus hijos... es decir... 'Bueno, usted aquí ya...', ino! 'Que estoy aquí, que le voy a echar una mano, que lo que usted necesite, que no pasa nada', y dicen 'Madre, me tiene que duchar!', pues ¡claro! y tendré que duchar a mi madre y yo también me ducho y tenemos lo mismo... es decir, que no sea esto un...mmm... ¿no?, un tabú, que no lo pase mal... (...) tenemos personas que han tenido que venir a solicitar algún papel donde la trabajadora social y es un hombre. ¿Por qué?, porque él es el viudo, ¿eh?, no tiene más remedio. Porque tiene hijos pero a lo mejor están trabajando. 'Pues, vengo aquí a traer unos papeles que los hijos como no están', está bien, que salgan y que vengan y que hablen... es decir, que rompan... mmm... aquí la gente $\mathrm{mmm} . .$. antes por lo menos, ¿no?, era muy... 'Madre, tengo que venir a hablar con esta muchacha [la trabajadora social]'. ¿Y qué pasa? ¡No pasa na’! Entonces era como... no sé... este ambiente de... como de vergüenza. No sé, ahora bastante bien. No, no, no, ¿eh? ¡Se han soltado! No, no, viene bien; es verdad" (trabajadora servicios públicos).

Ahora nadie cuestiona dichos servicios, porque ya no representa un estigma beneficiarse de estos (Bazo 1993), ni se cuestiona la gente que acude a ellos. Es como un círculo: más personas acuden a los servicios públicos, más legitimidad adquieren estos. Al mismo tiempo, más legitimidad alcanzan, más personas mayores los utilizan.

Este fenómeno se encuentra en los dos municipios de estudio, porque en otros pueblos cercanos, como revela la información recopiladas, esta legitimación todavía no ha tenido lugar, tanto que se ha llegado a suprimir algunos servicios públicos.

"Aquí la gente es menos reticente, pero hay otros pueblos... en Colmenar por ejemplo, seee... había Ayuda a domicilio... tuvimos que quitarla porque la gente se daba de baja porque... mmm... más que... quizás la persona en sí, LA FAMILIA. Decían 'Hombre, es que va a ir ALGUIEN, AJENO, va a entrar en casa, con lo cual me estás diciendo que yo soy una cochina'. Si nadie dice eso, si... mmm... tu puedes ir a casa de tu madre o tu padre a hacer lo que sea...' 'Sí sí, pero ¿y qué van a decir?'. Luego... mmm... las auxiliares nunca jamás encajan en Colmenar.... nunca jamás gusta la persona aunque sea... trabajadora, encantadora, amable y respetuosa, NUNCA jamás encajan. Entonces, Colmenar por ejemplo no tiene Ayuda a domicilio. ¡Claro!, no había demanda... por... desapareció. Y hay MUCHA GENTE en Colmenar que necesita Ayuda a domicilio. (...) además rechazan la ayuda, pero al final el mayor ¡no está atendido!... Al final el mayor no está atendido (...). De eso hay... aquí menos. Perooo... en Alcantara, por ejemplo, ha habido varios casos de eso. Ahora mismo hay uno... y no hay nada que hacer con él. De estos reticentes que no quieren ir a ninguna parte, que viven en unas condiciones lamentables, que no... no te dejan ni siquiera entrar en 
casa... que de aquellas casas se abre la puerta y sale... pffff... sapos y culebras... de esos hombres, de esos hay... y hay casos, ¿eh?" (trabajadora servicios públicos).

Este testimonio expresa la complejidad existente entre cuidados familiares y cuidados institucionales y entre los valores arraigados y los más actuales. Por otra parte, muestra cómo, en mayor o menor medida, la institucionalización del cuidado y su legitimación están modificando valores y acciones con la consecuente formación dinámica de unos nuevos modelos culturales.

\section{Conclusiones}

El principal resultado del estudio es la visibilización de un modelo circular donde la implantación de los servicios públicos de cuidado se configura como consecuencia de los cambios socio-demográfico de las localidades y, a su vez, como causa de los cambios en las relaciones sociales y en los valores del cuidado, en una amplitud de espacios y relaciones de cuidado (Del Valle 2004). Por tanto, la investigación por un lado revela la fuerte presencia de servicios públicos de cuidado en un espacio rural e indica que las zonas rurales no son todas "discapacitantes" por falta de servicios, como propugnan algunas investigaciones (Rodríguez Rodríguez 2004, Hernández 2002). Por otro lado, revela que no se trata solo de una aceptación o de un fracaso de los servicios públicos de cuidado, ya que, como hemos visto, no todos los servicios reciben la misma consideración y la misma legitimidad. Se trata más bien de ver cómo los servicios de atención para los mayores se han introducido en un contexto cultural y socioeconómico especifico, sirviéndose además de su característica cercanía relacional y espacial para diseñar unas acciones más acordes a las necesidades de las personas mayores y de sus familias, a beneficio de los individuos y de la colectividad. Esta característica, que funde en el cuidado formal y público las dimensiones del caring for y del caring about, representa la ventaja que tienen las zonas rurales para conseguir el éxito de los servicios públicos en la instauración de un modelo de intervención en red. En efecto, en las localidades se encuentran unas redes de cuidados, en las que se entrelazan los servicios directos ofrecidos por las instituciones, los servicios de la Ley de Dependencia, los cuidados informales de familiares, etc. constituye una superación del rígido binomio público/privado en el ámbito del cuidado (Bazo 2008, Tronto 2005 y 2010) y demuestra que el cuidado, como experiencia de reproducción social necesaria para el desarrollo de los individuos y de las sociedades, es una responsabilidad social que involucra a toda la sociedad (Cerri y Alamillo 2012).

Podemos hablar de un modelo de intervención en red (Vega Solís 2009) o de Social care (Martín Palomo 2008a, 2008b, 2009) que, por un lado, pretende que el Estado asuma su responsabilidad en cuanto proveedor de servicios de cuidado y, por otro, incluye todos los demás agentes de bienestar (el mercado, el voluntariado, la familia, los individuos) en una repartición de responsabilidad. De esta manera, el cuidado sale simbólicamente y materialmente del espacio doméstico y femenino, deja de ser una carga obligatoria y casi explotadora para las mujeres sin, por otra parte, abogar a la individualización familiar.

Para terminar, es innegable que el proceso de envejecimiento ha influido en la realidad social, económica y política de las localidades rurales introduciendo nuevas cuestiones. No obstante, estas no representan un obstáculo para el desarrollo socio-económicos y la formación de redes nacionales e internacionales. En efecto, los municipios de estudio no reproducen la típica imagen de "pueblos rurales": aislados de los procesos globales, caracterizados por valores y modos de vida tradicionales, poco tecnológicos, atrasados, etc. Al contrario, aunque algunos aspectos "tradicionales" se conservan (cuidar de la huerta y de los animales, participar en las fiestas locales, mantener distinciones más marcadas de espacios y roles en base al género, etc.), en los últimos años por ejemplo se ha trabajado para conseguir una mayor igualdad de género y se ha fomentado el uso de las nuevas tecnologías a través de cursos de informática (también para mayores), la instalación de redes Wifi, la creación de Telecentros, la Teleasistencia, la prescripción telemática de las recetas médicas en las farmacias, etc., que permiten una constante conexión entre lo local y lo global.

En este contexto, es posible afirmar que la presencia en las zonas rurales de los servicios públicos de 
cuidado, asistenciales y de envejecimiento activo, muestra los lazos existentes entre el mundo rural y el urbano, por lo que es difícil seguir afirmando que el campo y la ciudad están en posiciones totalmente opuestas. Sería más conveniente definir la realidad rural desde las relaciones que mantiene con otros sistemas y considerar así la conexión o desconexión entre el contexto local/rural y la sociedad global. Hablar a lo mejor de un "rural-urban continuum", como hace Ceña Delgado (1992, citado por García Sanz 1999) o de un "middle rurban ground" (Olsson y Ruotsala 2009) en el que los espacios rurales y urbanos se encuentran y el paisaje rural es cada vez más integrado en el medio urbano.

\section{Notas}

1. Trabajo realizado a través de la Ayuda FPI BES-2009-026934 en el Centro de Ciencias Humanas y Sociales (CCHS) del CSIC.

2. Los nombres reales de las personas, de los municipios y sus lugares han sido ocultados o sustituidos con nombres ficticios, para salvaguardar la confidencialidad de los informantes.

3. Tronto (2010) añade otras dos fases a las dimensiones del cuidado, para mostrar su más compleja realidad. Por una parte el care giving, "cuidar" y realizar los trabajos de cuidado. Por otro, el care receiving, "recibir cuidado". Cada fase está asociada a unas cualidades morales, que conllevan unos sentimientos y emociones: atención y consideración en el caring about, responsabilidad en el caring for, competencia en el care giving y, por último, responsividad (responsiveness) en el care receiving. El concepto de responsividad se ha desarrollado en la filosofía moral para reflexionar acerca de la relación entre las demandas y peticiones del cuidado (Patiño 2010).

4. El sistema "rotatorio" es viable en la Ayuda a domicilio porque los ayuntamientos son los únicos gestores y responsables. La realidad es distinta en el caso de las Residencias y los Pisos Tutelados donde existe una gestión privada, a través de un convenio con el Ayuntamiento.

5. Las políticas de envejecimiento activo promueven el uso de los potenciales físicos, sociales, mentales de todas las personas para fomentar su bienestar durante toda la vida y poder así participar en la sociedad. Al mismo tiempo, promueven una protección adecuada de seguridad y de atención cuando las personas lo necesiten.

http://www.who.int/ageing/active_ageing/en/index.html.

6. En su estudio sobre el fenómeno de las cuadrillas de amigos en la Comunidad Valenciana, Ricardo Sanmartín Arce (1999) sostiene que el acceso de los actores a la vecindad no tiene la misma libertad que la formación de la familia y de la amistad, porque los vecinos no siempre pueden ser elegidos. Por lo tanto, aunque la vecindad puede ser también fuente de amistad, es necesario que existan otros condicionantes para que así se formule.

\section{Bibliografía}

Alberdi Collantes, Juan Cruz

2007 "Contribución de la mujer al cuidado de los mayores residentes en el caserío: el caso de Oiartzun (Guipúzcoa)”, Ager, no 7: 61-100. 
Albert".

Barfield, Thomas (eds.)

2001 Diccionario de Antropología. Barcelona, Edicions Bellaterra.

Bazo, María Teresa

1993 "Mujer, ancianidad y sociedad", Revista Española de Geriatría y Gerontología, n 28, 1: 17-22.

2008 "Personas mayores y solidaridad familiar", Política y Sociedad, nº 45, 2: 73-85.

Boffil, Sílvia

2006 "Cuidado formal e informal en Cataluña. Un análisis de la percepción de las mujeres mayores en relación a sus derechos y obligaciones en materia de cuidado y atención”, Etnográfica, nº X, 2: 263-281.

Ceña Delgado, Felisa

1992 "Transformaciones del mundo rural y políticas agrarias", Revista de Estudios Agrosociales, n 162: 11-35.

\section{Cerri, Chiara}

2011a "De la 'buena vecina' a la cuidadora institucional. Los servicios públicos de cuidado como elemento de cambio en las relaciones vecinales entre mayores", en María Ángeles Durán (dir.), El trabajo del cuidado en América Latina y España. Madrid, Fundación Carolina - CeALCl: 149-152.

2011b "El cuerpo en la vejez: discursos y representaciones en el cuidado de las personas mayores en una zona rural de Extremadura", en Actas del XII Congreso Antropología FAAEE Lugares, tiempos, memorias. La antropología ibérica en el siglo XXI". León, FAAEE.

Cerri, Chiara (y Laura Alamillo-Martínez)

2012 "La organización de los cuidados, más allá de la dicotomía entre esfera pública y esfera privada", Gazeta de Antropología, no 28 (2), art. 14.

http://www.gazeta-antropologia.es/?p=4145

Comas d'Argemir, Dolors

1993 "Sobre el apoyo y el cuidado. División del trabajo, género y parentesco", en Roigé i Ventura, X. (coord.), Perspectivas en el estudio del parentesco y la familia. Tenerife, VI Congreso de Antropología: 65-82.

Comas d'Argemir, Dolors (y Jordi Roca)

1996 "El cuidado y la asistencia como ámbito de expresión de la tensión entre biología y cultura", en J. Contreras (coord.), Reciprocidad, cooperación y organización comunal. Desde Costa hasta nuestros días. Zaragoza, VII Congreso de Antropología: 57-69.

Canals, Josep

1996 "La reciprocidad y las formas actuales de ayuda mutua", en J. Contreras (coord.), Reciprocidad, cooperación y organización comunal. Desde Costa hasta nuestros días. Zaragoza, VII Congreso de Antropología: 43-55.

Cucó, Josepa

1995 La amistad: Perspectiva antropológica. Barcelona, Icaria.

De Haro, Alejandro

2011 "La ética del cuidado entre las personas mayores. Un estudio etnográfico en una institución residencial", Gazeta de Antropología, nº 27 (1), art. 1.

http://www.gazeta-antropologia.es/?p=1302

Del Valle, Teresa

2004 "Contenidos y significados de nuevas formas de cuidado", en EMAKUNDE/Instituto Vasco de la Mujer, Congreso Internacional Sare 2003: Cuidar cuesta: costes y beneficios del cuidado. Vitoria, 
Instituto Vasco de la Mujer: 39-61.

Durán, María Ángeles

2002 Los costes invisibles de la enfermedad. Bilbao, Fundación BBVA.

García Sanz, Benjamín

1999 La sociedad rural ante el siglo XXI. Madrid, Ministerio de Agricultura, Pesca y Alimentación, Secretaría General Técnica.

Hernández, Ángel

2002 "El envejecimiento de Castilla y León en el contexto español", en A. Blanco (ed.), Castilla y León. Envejecimiento y mundo rural. Madrid, Fundación Encuentro: 63-109.

INE

2001 Censo de población y vivienda 2001. Madrid, Instituto Nacional de Estadística.

2010 Padrón municipal 2010. Madrid, Instituto Nacional de Estadística.

Lisón Tolosana, Carmelo

2010 Qué es ser hombre (valores cívicos y valores conflictivos en la Galicia profunda). Madrid, Akal.

Martín Palomo, María Teresa

2008a "Los cuidados y las mujeres en las familias", Política ySociedad, n45, 2: 29-47.

2008b "Domesticar el trabajo: una reflexión a partir de los cuidados", Cuadernos de relaciones laborales, $\mathrm{n}^{\circ} 26,2: 13-44$.

2009 "El care, un debate abierto: de las políticas de tiempos al social care", Cuestiones de género: de la igualdad a la diferencia, nº4: 325-355.

Massone Moya, Francisca (y otros)

2010 " Observaciones de la vejez en familia. Una aproximación desde la corresidencia intergeneracional", Gazeta de Antropología, nº 26 (1), art. 17.

http://www.gazeta-antropologia.es/?p=1685

Mauss, Marcel

1950 Sociología y Antropología. Madrid, Editorial Tecnos, 1971.

Narotzky, Susana

2009 "Trabajo a domicilio y trabajo doméstico en la globalización neoliberal. Del feminismo igualitarista a la ética del cuidado: situando responsabilidad", en A. Téllez Infantes y J. Martínez Guirao (eds.), Economía informal y perspectivas de género en contextos de trabajo. Barcelona, Icaria: 197-217.

Olsson, Pia (y Helena Ruotsala) (eds.)

2009 Gendered rural spaces. Helsinki, Finnish Literature Society.

Patiño, Susana Magdalena

2010 La responsividad ética. Madrid, Plaza y Valdés.

Pérez Díaz, Julio

2005 "Consecuencias sociales del envejecimiento demográfico", Papeles de Economía Española, n 104: 210-226.

2006 “Demografía y envejecimiento", Informes Portal Mayores, 51. Lecciones de Gerontología, I .

http://www.imsersomayores.csic.es/documentos/documentos/perez-demografia-01.pdf

Rodríguez Rodríguez, Pilar

2004 "Envejecimiento en el mundo rural: necesidades singulares, políticas específicas", Perfiles y Tendencias 11, Madrid: Instituto de Mayores y Servicios Sociales.

Rogero-García, Jesús 
2009 "La distribución en España del cuidado formal e informal a las personas de 65 y más años en situación de dependencia", Revista Española de Salud Pública, nº 83: 393-405.

2010 Los tiempos del cuidado. El impacto de la dependencia de los mayores en la vida cotidiana de sus cuidadores. Madrid, Instituto de Mayores y Servicios Sociales (IMSERSO).

Sahlins, Marshall

1974 "Economía tribal", en M. Godelier (coord.), Antropología y Economía. Barcelona, Anagrama, 1976: 233-259.

Sanmartín Arce, Ricardo

1999 Valores culturales. El cambio social entre la tradición y la modernidad. Granada, Editorial Comares. 2003 Observar escuchar comparar escribir. La práctica de la investigación cualitativa. Barcelona, Editorial Ariel.

Tobío, Constanza (y otras)

2010 El cuidado de las personas. Un reto para el siglo XXI. Barcelona, Fundación "La Caixa".

Tronto, Joan

1987 "Beyond gender difference to a theory of care", Signs, n 12, 4: 644-663.

2005 "Cuando la ciudadanía se cuida: una paradoja neoliberal del bienestar y la desigualdad", en EMAKUNDE/Instituto Vasco de la Mujer, Congreso Internacional Sare 2004 ¿Hacia qué modelo de ciudadanía? Vitoria, Instituto Vasco de la Mujer: 231-253.

2010 "Cura e politica democratica. Alcune premesse fondamentali", La societá degli individui, n 38, 2: 34-42.

Vega Solís, Cristina

2009 Culturas del cuidado en transición. Barcelona, Editorial UOC. 